\title{
Sobre la literaturización de las ciencias sociales o cómo las teorías se convierten en débiles. Una crítica a los estudios culturales
}

Andrea Marina D'Atri ${ }^{1}$

\section{RESUMEN}

La propuesta de este artículo es analizar el posicionamiento de los estudios culturales en América Latina como teoría de conocimiento y la valoración de su potencia crítica a partir de algunos autores actuales. Teorías débiles, o de mínimo compromiso con lo epistemológico y una actitud conciliatoria con el poder dominante en lo ideológico, versus teorías fuertes, aquellas en que la potencia crítica y la exigencia epistemológica perdura, es la dicotomía que atraviesa las discusiones al respecto y que propone el investigador argentino Roberto Follari. De este modo, desde una mirada comprensiva de diversos textos, nos enfocamos en el problema que implica lo que denominamos una "literaturización" de las ciencias sociales.

Palabras claves: Literaturización, ciencias sociales, teorías débiles, estudios culturales.

1 Departamento de Comunicación Social, Facultad de Ciencias Humanas, Universidad Nacional de La Pampa. Mail de contacto: andreadatri2012@gmail.com 


\begin{abstract}
The proposal of this article is to analyze the positioning of cultural studies in Latin America as a theory of knowledge and the assessment of its critical power from current authors. Weak theories, or of minimal commitment to the epistemological and a conciliatory attitude with the dominant power in the ideological, versus strong theories, those in which the critical power and the epistemological demand persists, is the dichotomy that crosses the discussions on the matter and proposes the Argentine researcher Roberto Follari. In this way, from a comprensive perspective of various texts, we focus on the problem that involves what we call a "literaturization" of the social sciences.
\end{abstract}

Key words: Literaturization, social sciences, weak theories, cultural studies

Fecha de recepción: 23/09/2019

Fecha de aceptación: 26/06/2020 


\section{Sobre la literaturización de las ciencias sociales o cómo las teorías se convierten en débiles. Una crítica a los estudios culturales}

\section{INTRODUCCIÓN}

La literaturización ${ }^{2}$ de las ciencias es nuestro punto de arranque, con el fin de introducirnos en el sustento teórico desde el cual parten los estudios culturales, es decir, desde su objeto de estudio intentando apartarse de las explicaciones que da el marxismo. Con este propósito, en este artículos nos proponemos desde una lectura hermenéutica, poner en situación de diálogo e interpretar un texto de Roberto Follari (2002) con otro de Stuart Hall (2017), ya que ambos consideran -aunque de diferente modo y en diverso ámbito- el problema que implica la "literaturización" de las ciencias sociales; el primero, en referencia a cómo los cultural studies del Hemisferio Norte se asientan en Latinoamérica (Follari, 2002, p. 8) y, el segundo, al desarrollar en una conferencia sobre el culturalismo y los aportes de Raymond Williams, la definición que ese teórico inglés efectúa sobre la cultura. Allí, en Cultura y Sociedad (Williams, 1958/2001), dice Hall que al autor "el foco literario no lo abandona" y se presenta la dificultad que se le hace evidente:

...reconocer que la problemática de la cultura le exige explorar cómo poder abordarse las producciones literaria y artística en una perspectiva social y cultural, antes que en los términos puramente estéticos y morales de los que se apropiaron previamente Leavis y sus seguidores. (...) Aun cuando lucha por escapar de ella, Williams continúa siendo parte de la tradición... (Hall, 2017, p. 55).

El interrogante, para comprender el valor de las teorías, es preguntarse desde qué posición se va a analizar la sociedad. En este sentido, el marxismo constituiría la teoría fuerte que sirve de anclaje, ya que es la perspectiva que puede dar respuesta para un análisis que no quede meramente en lo discursivo, literario o narrativo. Es decir que interesa saber si nos quedamos o no en un análisis de los procesos que incorpore las dimensiones del poder; y de un poder vinculado a lo político (y no únicamente con respecto a las identidades y las textualidades).

Un glosario para comprender la dicotomía a la que hacemos referencia, entonces, debería iniciarse definiendo qué son los estudios culturales, seguir por la literaturización como parte de éstos -y, en su marco, analizar qué son desde la posmodernidad- para llegar a una enunciación que dé cuenta de la construcción de campos epistemológicos 'fuertes' o 'débiles'. Los términos y sus significaciones se integran por completo dado que, como cuando se estudian procesos identitarios, no puede concebirse una fuerza sin su oponente o una identidad sin un 'otro'. Con esto, queremos decir que analizaremos las tensiones entre teorías más o menos fuertes según los autores mencionados, a partir de observar cómo los llamados estudios culturales consideran la cultura -resignificando que no se trata simplemente de una determinación

2 Lo veremos más adelante, pero diremos que por literaturización, Follari (2001) entiende una generalizada proliferación de retórica por sobre el análisis empírico, así como una "libre reflexión" por sobre las exigencias de las teorías científicas. 
de lo estructural sobre lo superestructural- pero, en algunos casos, implicando una discursividad que la lleva a inscribirse en una epistemología débil.

\section{CULTURAL STUDIES Y DESPLAZAMIENTOS}

Inglaterra, década de 1950. En ese lugar y en ese momento tienen origen los denominados cultural studies. Hall (2017) se pregunta y expone el contexto social y político que se vive en la Gran Bretaña de las décadas posteriores a la Segunda Guerra Mundial, especialmente los años cincuenta y sesenta, un período de un nivel de opulencia económica inusualmente alto y un crecimiento sostenido "como la economía del Reino Unido no había visto en todo el siglo XX" (Hall, 2017: p. 28):

Es importante comprender que el concepto de cultura se propuso no como la respuesta a alguna gran pregunta teorética sino como una respuesta a un problema político y un interrogante muy concreto: ¿Qué pasó con la clase trabajadora a partir del advenimiento de la abundancia económica? (Hall, 2017: p. 28).

En este período, comienzan a publicarse una serie de investigaciones de autores cuyo punto de contacto es ser parte de una generación que comparte la experiencia común de la posguerra; de ser hijos de la clase obrera británica. Es el caso de Raymond Williams y Richard Hoggart, que provienen de la literatura.

Hoggart escribe en 1957 Los usos de la alfabetización (Hoggart, 2013), cuyo tema de análisis es la cultura obrera del pobre, una suerte de texto antropológico donde describe los rasgos de la cultura de este grupo social británico, sus formas de sociabilidad, el modo de ser de las familias, entre otros aspectos. También formula la pregunta acerca de si el desarrollo de la industria cultural -por tanto, de los medios masivos- no va a terminar borrando las características de la cultura obrera con sus tradiciones. Y, al final, inquiere si no estará amenazada la cultura obrera por 'una cultura sin rostro' o aquella comunicación masiva de mediados del siglo XX que pretende una cultura sin clase (Hoggart, 2013).

Por su parte, Raymond Williams, con una biografía similar, afiliado durante un año al Partido Comunista, accede a la universidad y será docente de escuela de adultos y luego de la misma alta casa de estudios donde se forma (Trinity College, Cambridge). En 1961 publica La larga revolución y, en 1970, Marxismo y literatura. Williams dice que cultura es lo 'ordinario', lo común, y combate la idea de cultura que tiene el marxismo, en la cual prima el determinismo de la estructura sobre la superestructura. El autor piensa la sociedad según tres conceptos claves: hegemonía (a partir de las lecturas sobre este concepto de Gramsci (1998), cultura y experiencia. La cultura se conforma a partir de las experiencias. Dirá Hall (2017) que para Williams cualquier práctica conlleva una cultura. Permítasenos esta extensa cita del primer autor mencionado:

Los estudios culturales procuran encontrar las formas comunes de experiencia y las definiciones compartidas que vive una comunidad. Por lo tanto, los estudios culturales no son una descripción objetiva de cómo vive un grupo ni un análisis o inventario de las ideas que ese grupo tiene. Puesto que la cultura es la interacción entre ambas cosas, los estudios culturales exploran la vida que el individuo está obligado a vivir a causa de las condiciones en las cuales ha nacido, las circunstancias que le han sido presentadas 
como significativas $y$, por ende, dignas de experimentarse, porque a esas condiciones se les han aplicado ciertos marcos de comprensión [...]. Esas formas de experimentar se han incorporado a las maneras de vivir de los seres humanos, a sus prácticas. Una práctica siempre conlleva una cultura. Ha sido cultivada. Está impregnada de formas de interpretación. Eso es la cultura: experiencia vivida, experiencia interpretada, experiencia definida. Y esto es lo que Williams Ilama experiencia. (Hall, 2017: p. 60)

Las preguntas fundamentales que se hacen la mayor parte del tiempo desde los Estudios Culturales ingleses -que van a institucionalizarse como tales a partir de la obra de Hoggart (1957/2013)- atraviesan la cultura y, en su escenario, las experiencias que los sujetos ponen en juego, es decir sus prácticas sociales. En ese sentido, desde este marco conceptual se analizarán por ejemplo, las audiencias de la industria cultural. Y es considerando justamente esta industria, que en su célebre texto Encoding/ Decoding, Hall (1973) quiere refutar los postulados funcionalistas y enfoca -desde los estudios de la recepción- el proceso de comunicación audiovisual según cuatro procesos: producción, circulación, distribución/consumo y reproducción, en relación con determinaciones que implican relaciones de poder institucionales. Hall postula que la audiencia es receptora y fuente del mensaje al mismo tiempo, porque los esquemas de producción responden a las imágenes que la institución televisual se hace de la audiencia. Para el autor, importa en particular la audiencia porque ésta puede decodificar de distinto modo lo que recibe: de manera dominante, de manera negociada o de manera oposicional. El autor realiza esta clasificación a partir de la teoría de los sistemas de sentido desarrollada por Frank Parkin (1972), quien explica, según retomamos de O'Sullivan et al., (1995), las diversas respuestas de los grupos a sus diferentes condiciones sociales, o cómo las personas dan sentido a su mundo social. Parkin, a la vez, identifica tres principales sistemas de sentido en las democracias industriales occidentales:

i. Sistema dominante: que confirma la estructura existente de relaciones sociales, económicas y políticas y permite que la gente entienda cuál es su posición social dentro de la distribución de poder, riqueza y trabajo establecida;

ii. Sistema subordinado: que acepta el sistema general dominante, pero permite que grupos particulares tengan el derecho de demandar una posición mejor dentro de él;

iii. Sistema radical: rechaza el sistema dominante y propone otro diferente opuesto a aquel (O'Sullivan et al., 1995: p. 336-337).

Hall sostiene que estos sistemas de significación establecidos en la sociedad, que no son recíprocamente excluyentes, son el medio a través del cual las personas hacen que su contexto social adquiera peso en la decodificación de los textos televisivos o de otros medios de difusión masiva. Los sistemas producen tres códigos equivalentes para decodificar o leer el texto: el hegemónico o dominante, el negociado o el de oposición.

En América Latina, investigadores como Jesús Martín Barbero desde Colombia; Beatriz Sarlo desde Argentina; Guillermo Sunkel desde Chile o Néstor García Canclini -argentino radicado en México-, orientan sus lecturas y producciones en el sentido de los estudios culturales ingleses. En un anterior trabajo (D'Atri, 2004) decíamos que las 
teorías de la recepción ${ }^{3}$ en América Latina se sostienen en los análisis de la corriente que se despliega en las décadas del sesenta y setenta del siglo pasado bajo el nombre de Estudios Culturales, que luego se enriquecen con el interaccionismo simbólico de la escuela de Chicago (en su análisis interpretativo de lo social y desde la perspectiva de los participantes) y con la renovación de la tradición etnográfica británica (cultural studies).

En De los medios a las mediaciones. Comunicación, cultura y hegemonía (1987), Jesús Martín Barbero propone "desplazarnos del espacio de los medios al lugar en que se produce su sentido" (Barbero, 1987: p. 213), corrernos de los medios, hacia las mediaciones, entendidas como aquellas zonas donde se articulan la producción de significados de las clases dominantes y la producción de significados de las clases subalternas. Propone conocer las matrices culturales que articulan zonas de producción de sentidos.

De manera que para los investigadores latinoamericanos, el estudio de la sociedad y la cultura se va a efectuar desde dimensiones que comprenden la estructuración socioeconómica, las identidades culturales, las definiciones del espacio público y las redefiniciones posmodernas. Hay un desplazamiento del estudio de la recepción, hacia una etnografía de la cultura (Canclini, 1990) que busca conocer los diversos componentes de la trama social. García Canclini (1988) va a estudiar el modo como se produce el consumo, ese espacio en el que se forman las identidades colectivas y las diferencias de clases. En sociedades que se pretenden democráticas, analiza aquella producción (el consumo) como el lugar que construye y comunica las diferencias (qué y cómo consumimos, ayuda a diferenciarnos):

El consumo también se considera un sistema de integración y comunicación, porque debe ser un sistema comprensible que dé sentido a las diferencias. Además, implica un lugar de objetivación de los deseos, un deseo sin objeto, errático e insaciable (Canclini y Roncagliolo, 1998, p. 51).

Dicen Canclini y Roncagliolo (1998) que, mientras algunos autores definen el consumo sólo como el lugar de reproducción de la fuerza de trabajo y expansión del capital, otros agregan a este postulado aquel que lo define como un espacio donde las clases luchan por la apropiación del producto social, ya que hay mayor cantidad de objetos, mayor circulación y consumo como resultado del crecimiento de las demandas y de las luchas populares.

\section{3. "PÉRDIDA IDEOLÓGICA”}

Al considerar los estudios culturales en Latinoamérica, el investigador argentino Roberto Follari (2002) observa que hay que tener en cuenta que en nuestros países "se hablaba de cultura desde antes, pero se lo hacía de otra manera" (Follari, 2002, p.

3 Se trata de distintas propuestas teórico-metodológicas que se presentan contra las grandes referencias que dominaron por décadas el conocimiento de la comunicación en las sociedades, es decir, tanto contra el funcionalismo como contra el marxismo. Investigadores como Guillermo Orozco Gómez, Mercedes Charles Creel, Valerio Fuenzalida y María Helena Hermosillo -en las corrientes que se refieren a la recepción activao aquellos de Jesús Martín Barbero, Jorge González, Néstor García Canclini, Armand y Michele Mattelart y Mabel Piccini, en los estudios de la recepción en la trama de la cultura (D’Atri, 2004). 
56); que es preciso diferenciar los estudios culturales de los estudios poscoloniales 4 . Afirma, asimismo, que caracteriza a los estudios culturales una pérdida del perfil ideológico y de actitud subvertora o de transformación, en consonancia con las tendencias de la posmodernización cultural y con el poder académico institucionalizado. En este sentido, las críticas hacia los estudios culturales latinoamericanos de parte del autor argentino, se sustentan en la pérdida o 'debilitamiento ideológico' de quienes suscriben este marco de análisis, y cita a Sarlo (1996), Barbero (1987) y Canclini (1990; 1995), así como a H. Achúgar (1999), quien también se ocupa del tema desde Uruguay.

La dificultad que se presenta en el anclaje de los estudios culturales en Latinoamérica, según Follari (2002), se refiere a la propuesta metodológica y epistémica de la misma. El objeto de análisis, tanto de la deconstrucción como de los estudios culturales, es a la vez su 'síntoma'. Dice el autor: "deconstrucción y cultural studies participan en común de un nuevo y pregnante proceso que desde la academia del hemisferio norte se está asentando vigorosamente en Latinoamérica: la progresiva literaturización de las ciencias sociales" (Follari, 2002: 8).

De igual modo pero en otro contexto se había edificado una 'metodología' donde lo literario y lo textual se inmiscuyen para analizar la cultura: tanto Williams como Hoggart fueron construyendo de esa manera lo que luego sería el sustento epistemológico de los cultural studies.

Hoggart aplica una especie de imaginación literaria al análisis de una cultura. Y, si bien se sitúa dentro del linaje cultural literario tradicional, también se ubica fuera de él, porque esa tradición nunca consideró que ese tipo de cosas sobre las que escribe merecieran la más mínima atención (Hall, 2017: p. 34).

El problema, advierte Follari -y no es respuesta a lo anterior porque el objeto de estudio se sitúa en nuestros países latinoamericanos, si bien consideramos que bien vale el diálogo ficticio-, es que se ha originado esta especie de "proliferación generalizada de la retórica por sobre el análisis empírico, y de la libre reflexión por sobre las constricciones y exigencias de las teorías científicas" (Follari, 2001: p. 8). Los estudios culturales latinoamericanos pierden sus enclaves tanto empíricos como materiales. Agrega el investigador argentino:

Desaparece lo socioeconómico, en aras de lo cultural o de lo lingüístico. Todo se transforma en textual, y en consecuencia aún las relaciones de propiedad e interés económico sobre las que se establece la circulación de información, se borran casi por completo de los análisis. El tema del poder se va evaporando (...) La política brilla por su ausencia (...) Cuanto más necesita Latinoamérica apoyo conceptual para la constitución de pensamiento crítico en esta época de dictadura generalizada de los mercados, menos ayudan estos discursos a establecerlo. (Follari, 2002: p. 9).

4 Desde las investigaciones del peruano Aníbal Quijano en torno a la colonialidad del poder, se articulan a fines de la década de los noventa del siglo XX un conjunto de estudios que trabajan problemas teóricos y sociales que se pensaban clausuradas en las ciencias sociales latinoamericanas. Para el grupo GESCO (2012) de estudios decoloniales, “la revisión de la constitución histórica de la modernidad y sus transformaciones en América Latina ha sido el nodo desde el cual se han articulado estas problemáticas, a la luz de la categoría de colonialidad como la contracara de la modernidad". 


\section{MÁS DICOTOMÍAS}

Nos interrogamos, así, acerca del modo en que los autores referentes de los estudios culturales latinoamericanos desplazaron de sus análisis la mirada sobre el soporte material y empírico de sus objetos de estudio.

Canclini, al final del texto De cómo Clifford Geertz y Pierre Bourdieu Ilegaron al exilio (1998), se refiere al objeto de estudio de las ciencias sociales como el de los cruces interculturales, y menciona la necesidad de "salir del ensimismamiento de las disciplinas y las instituciones de su reorganización acrítica bajo el mercado" (Canclini, 1998: p. 39). ¿Pero da pistas concretas acerca de cómo hacerlo? Dice el autor:

En un tiempo de globalización, el objeto de estudio más revelador, más cuestionador de las pseudocertezas etnocéntricas o disciplinarias, es la interculturalidad. El científico social puede, mediante la investigación empírica de relaciones interculturales y la crítica autorreflexiva de las fortalezas disciplinarias, intentar pensar ahora desde el exilio. Estudiar la cultura requiere, entonces, convertirse en un especialista de las intersecciones (Canclini, 1998: p. 39).

El autor postula que existen culturas populares porque la reproducción desigual de la sociedad genera una apropiación desigual de los bienes económicos y culturales por parte de diferentes clases y grupos en la producción y en el consumo. Agrega que se produce tanto una elaboración propia de sus condiciones de vida y una satisfacción específica de sus necesidades en los sectores excluidos de la participación plena en el producto social, como una interacción conflictiva entre las clases populares con las hegemónicas por la apropiación de los bienes. A esta teoría reproductivista (marxista), el autor le agrega otras teorías sociológicas, más atentas a los procesos desiguales de consumo y a la formación de consenso que hace aceptar esa desigualdad (Canclini, 1988).

Asimismo, en Cultura trasnacional y culturas populares (1988), analiza que el paso de la teoría de la dependencia o de la dominación ${ }^{5}$ a aquella que bajo la influencia gramsciana enfocaba el estudio de las culturas teniendo en cuenta las relaciones hegemonía-consenso, admitía "espacios donde los grupos subalternos desarrollan prácticas independientes y no siempre 'funcionales' para la reproducción del sistema" (Canclini, 1988: p. 55).

Pero las críticas a esta particular posición de Canclini tienen que ver con que el autor se mueve entre Bourdieu y Gramsci y los dos juntos no caben en un mismo discurso sobre cultura popular (Brunner, 1988): Brunner se pregunta qué tiene de popular la cultura hegemónica interiorizada por los sectores subalternos, y qué tiene de opuesto a la hegemonía (a lo dominante), la cultura popular, que es, a la vez, reproducción de esa hegemonía. "Si la sociedad es vista como la reproducción de una hegemonía, entonces no queda claro el papel que podría jugar en este proceso la noción de cultura popular", (Brunner, 1988: p. 82), dice el autor, dejando de lado a Bourdieu.

5 Sunkel y Paz (1975, El subdesarrollo latinoamericano y la teoría del desarrollo, México: Siglo XXI), postulan que son teorías en las que desarrollo y subdesarrollo son dos aspectos diferentes de un mismo proceso y por la que se pueden identificar distintas etapas en la historia de América Latina en términos de las relaciones de producción dominantes y la producción de pobreza. 
También Follari va a señalar las contradicciones y vaivenes de Canclini en sus postulados desde los estudios culturales, con respecto a la mirada de la dimensión del poder y a los estudios culturales propios. Expresa:

Hemos ya señalado suficientes críticas con relación a los últimos trabajos del autor argentino. Esperamos que -en conocimiento de su reconocida capacidad y de su trayectoria en torno a estudios sobre culturas populares- lo veamos en el futuro retomar las posibilidades interpretativas de los EC (estudios culturales), sin asumir sus liviandades epistemológicas y teóricas (Follari, 2002: p. 102).

Follari no oculta para nada su ofuscamiento ante la visión teórica y epistemológica 'liviana' que transmite y enuncia Canclini e ironiza acerca de sus posibles nuevos estudios sobre culturas populares.

\section{SI LO POLÍTICO ESTÁ AUSENTE}

Decíamos al inicio de este artículo que el problema que suscita la literaturización adherida a los estudios culturales es que se entrona en una discursividad con ausencia de marcos ideológicos y, en consecuencia, se los puede considerar 'débiles' desde el punto de vista teórico y epistemológico. Follari argumenta, en este orden, que el problema del abandono de lo político y la indefinición ideológica de los estudios culturales proviene justamente de la consideración de lo micro de estos últimos. Lo micro serían, por ejemplo, las 'intersecciones culturales' de las que habla Canclini. "Si bien lo micro es un dispositivo fundamental en la elaboración de hipótesis y conjeturas y, aún en la exploración de los conflictos estructurales, puede transformarse en una coartada cuando no es acompañado por lecturas del mismo objeto en otras escalas", arguye Follari (2002: p. 102).

Lo micro o lo no estructural; las identidades, sin estructuras de poder; las narrativas y los discursos, sin contextos políticos y económicos, son diversos modos de decir algo similar:

Esperamos respuestas más plausibles que las que hasta ahora venimos encontrando, cuando los autores de EC se preguntan 'desde dónde' hablan éstos. Se nos dice que 'desde los intersticios', pero no desde dónde se llega a estos. Ni de qué intersticios se trata. Y qué tienen que ver con la historia y la vida de los que escriben, y con la de aquellos otros a que refiere la escritura (Follari, 2002: p. 104).

La responsabilidad -en parte- de que esto haya ocurrido -o esté ocurriendo-, tanto en nuestros países como en los de nacimiento de los estudios culturales, se debe -según Follari- a la institucionalización académica universitaria. Como en todo su libro -Teorías débiles: para una crítica de la deconstrucción y de los estudios culturales (Follari, 2002)-, el autor intenta contraponer las teorías fuertes a otras no consistentes, pero, sobre todo, está observando qué elementos deberían contener unas teorías cuya epistemología perdure y tenga una potencia tal que permita el pensamiento crítico. Porque detenerse en el análisis de los procesos identitarios o culturales vinculados a los consumos culturales o, analizar otras manifestaciones de las industrias de comunicación de masas, para ejemplificar, no tendría sentido si no es en vinculación 
con las dimensiones del poder o la construcción social de esas identidades desde las políticas públicas, o poniendo en cuestión la misma construcción discursiva -pero llevada al campo de las teorías y las prácticas sociales (la enseñanza, por ejemplo)generada desde los espacios de poder de la academia (para el autor, la academia universitaria).

\section{EL SUR}

En este orden, otro autor del cual se ocupa el investigador argentino para interrogarse acerca del potencial crítico de las teorías, es el portugués Boaventura de Sousa Santos (2009), quien propone una epistemología del sur en su reconocido texto que lleva el mismo nombre. Y lo toma, justamente, porque desde el comienzo de su producción, de Sousa se mostró preocupado por la relación entre el conocimiento y la acción:

Se desprende de sus obras una rebeldía ante la escasez de uso del conocimiento producido, ante la producción que no tuviera en cuenta el uso, ante la incapacidad de las ciencias sociales para alumbrar modificaciones concretas en los espacios de la vida social (Follari, 2019: p. 126).

Si bien le va a criticar su "fuerte apuesta a la voluntad", Follari se detiene en de Sousa por cuanto éste pone en cuestión que lo posmoderno es una época de cierre a la noción de cambio de la historia y de caída de los relatos de revolución social. Dice:

Nos invita así BSS a la aventura intelectual de 'pensar sin el otro dicotómico', según solemos hacerlo: pensar el Sur como si no hubiera Norte, pensar la mujer como si no hubiera el hombre. Esa modalidad radicalmente antidialéctica del pensamiento, nos libraría de la atadura de someter siempre el acontecimiento a determinaciones que le fueran externas, de la incapacidad para pensar a los dominados en su singularidad, en vez de hacerlo siempre relacionándolos con las ataduras que los limitan desde quienes operan en el sector social hegemónico (Follari, 2019: p. 130).

Luego, va a señalar la 'audacia intelectual' de de Sousa, quien arriesga mediante una noción de totalidad, el análisis social: "Se advierte la intencionalidad de lo propuesto por BSS: liberar a la acción de esa rémora cuasi-religiosa que lleva a esperar siempre las condiciones estructurales del todo para emprender la acción sobre alguna de las 'partes'..." (Follari, 2019: p. 130).

La cita sirve para entender por qué para el autor argentino, el lusitano expondrá sus ecologías y, entre ellas, la de 'saberes' que ha tenido una amplia recepción positiva "en el campo académico que se reclama crítico, al menos el de Latinoamérica". En este sentido, observará la elaboración teórica de Boaventura de Sousa Santos -si bien no la considera una 'teoría débil'-, por cuanto no es posible una radical democracia epistémica a partir de armonizar posiciones diversas donde se legitimen saberes 'secundarizados'. Es decir, no es posible reducir las diferencias a una totalidad; de Sousa Santos supone un orden difícil de ordenar:

(...) supone una pretensión de superación del caos en dirección al orden, que nada en la realidad social contemporánea parece autorizar... Los saberes populares, los afros, los 
de diversas etnias indígenas, los de las ciencias, son mutuamente inconmensurables; y nada garantiza que, desde ellos, todos sus sujetos aspiren a la emancipación (Follari, 2019: p. 131).

¿Qué quiere decir el autor? Si relacionamos el análisis que Follari despliega sobre la propuesta de una epistemología del sur, con el problema que supone no vincular las teorías con las estructuras sociales desiguales, diríamos que al igual que hace con los estudios culturales, en el caso de la ecología de saberes de de Sousa, cuestiona la imposibilidad de un diálogo entre diversos saberes -con sus legitimidades intrínsecas- para la emancipación, ya que es una "noción idealizada y conciliatoria de diferencias que reclaman su diversificada atención y su exterioridad entre sí" (Follari, 2019: p. 132). Advierte:

La (imposible) armonía entre posiciones diversas que se reclama en la ecología de saberes, busca una legitimidad para los saberes secundarizados, y propone una radical democracia epistémica. Pero lo hace con una pretensión de reducción de la diferencia al Uno que es propia de lo más acendrado de la modernidad hegemónica de la cual, como hemos analizado más arriba, BSS busca salir con sus críticas a la noción de totalidad: y supone una pretensión de superación del caos en dirección al orden, que nada en la realidad social contemporánea parece autorizar (Follari, 2019: p. 132).

Al concluir su artículo, el investigador argentino redoblará su crítica sobre el mismo tema y dirá que en términos de la acción política emancipatoria, el autor portugués deposita su posibilidad en el diálogo de saberes de los movimientos sociales. Follari responde que no es justamente ese el objetivo que mueve a las distintas luchas de los movimientos, ya que "en la polivocidad de sus versiones reside la imposibilidad misma de la reducción a criterios en común, aun cuando se los quiera emancipatorios" (Follari, 2019: p. 133).

En resumen, entendemos que el autor descree de la posibilidad de que se armonicen (o dialoguen en el sentido de buscar acuerdos para la emancipación o ruptura de la desigualdad) y, por lo tanto, puedan legitimarse saberes populares como los mencionados en una "radical democracia emancipadora" totalizante, porque es intrínseco a aquellos su polivocidad. ¿Porque, además, quién dice que se pretende una única voz? ¿O quién estaría autorizado a decir qué voz darle a quién? A esto se refiere el autor cuando afirma que sería una "pretensión de reducción de la diferencia al Uno que es propia de lo más acendrado de la modernidad hegemónica".

\section{REFLEXIONES FINALES}

Si, por su lado, Stuart Hall revisa el origen de los cultural studies a través de un foco literario que no abandona a Raymond Williams al continuar una tradición de abordaje de lo social y cultural desde lo estético y moral, por otro lado, para nuestra gran región latinoamericana, Roberto Follari desmenuza la literaturización de las ciencias a través de un proceso en el cual la influencia del viraje al estudio de la cultura, las prácticas sociales y las identidades con la influencia de los estudios culturales tienen un rol preponderante. Follari lee autores latinoamericanos que desarrollan ideas provenientes de esta tradición (los cultural studies influenciados por su paso americano 
y que generan bibliografía), pero observa que no profundizan tanto como para transformar ese aparato crítico en una herramienta que analice y ponga al descubierto las tramas del poder. Por el contrario, se apartan de ellas. La literaturización de las ciencias sociales, de la historia, de las epistemologías, construye e instituye narrativas, por lo que se vuelven teorías 'débiles' que no logran interpelar a la política y al poder hegemónico como instituciones sociales transformadoras. No alcanza con mirar las tramas, los intersticios (Canclini, 1990), lo micro, las mediaciones (Barbero, 1987) desde la cultura, si no se dice cómo se articulan esas apropiaciones políticamente o en la acción política.

La relectura de autores que desarrollan una epistemología propia, como la de Boaventura de Sousa Santos, si bien contiene la intención de una 'radical democratización del conocimiento', se supondría insatisfactoria ya que desde un voluntarismo (o el acuerdo que propone un "diálogo de saberes") no se lograrán los objetivos de una transformación social emancipatoria.

Los debates no son nuevos ni es posible cerrarlos con sentencias. Las ciencias sociales y los científicos y científicas tenemos el enorme desafío de seguir pensando en pos de una dilucidación tanto teórica como metodológica desde nuestros espacios de prácticas, por unas epistemologías críticas que apunten a una transformación y que, con fortaleza, contribuyan a hacer plausible -pero más que todo, comprensibleel mundo social. La tarea sería dar pistas a través de teorías críticas que manifiesten y permitan comprender, para contribuir a revertir, las desigualdades presentes. 


\section{REFERENCIAS BIBLIOGRÁFICAS}

1. Achúgar, Hugo (1999). La incomprensible invisibilidad del ser económico, o acerca de cultura, valor y trabajo en América Latina. En García Canclini, N. y Moneta, J (coords.), Las industrias culturales en la integración latinoamericana, (pp. 309-322). Buenos Aires: Eudeba.

2.Barbero, J. (1987). De los medios a las mediaciones. Comunicación, cultura y hegemonía. Barcelona: Editorial G. Gili.

3. Boaventura de Sousa Santos. (2009). Epistemología del sur. México: Siglo XXI Editores.

4. Brunner, J. (1988). Políticas culturales en América Latina, en Cultura trasnacional y culturales populares. Buenos Aires: Colección Enlace, Grijalbo.

5. Canclini, G. N., Roncagliolo, R. (eds.). (1988). Cultura trasnacional y culturas populares. Lima: IPAL.

6. Canclini, N. (1988). De cómo Glifford Geertz y Pierre Bourdieu llegaron al exilio, en Causas y Azares. Los lenguajes de la comunicación y la cultura en (la) crisis, 7, pp. 26-40.

7.-------- (1990). Culturas híbridas. Estrategias para entrar y salir de la modernidad. Buenos Aires: Paidós.

8. ------- (1995). Consumidores y ciudadanos (conflictos culturales de la globalización). México: Grijalbo.

9. D'Atri, A. M. (2004). Con o sin textos de lecturas. Estudio sobre la recepción. Anuario, VI, 6, pp. 141-153.

10. Follari, R. (2002). Teorías débiles: para una crítica de la deconstrucción y de los estudios culturales. Rosario: Homo Sapiens.

11. ----- (2019). Boaventura de Sousa Santos: reducción de la dispersión al orden en la ecología de saberes. Utopía y praxis latinoamericana, 24, № 86 (julio-septiembre), pp.125-134.

12. Gramsci A. (1998).Cartas desde la cárcel. Traducción de Gabriela Moner para Editorial Lautaro, 1950. Buenos Aires: Ediciones Nueva Visión.

13. Grupo de Estudios sobre Colonialidad (Grupo GESCO). (2012). Estudios decoloniales: un panorama general, en Revista Kula. Antropólogos del Atlántico Sur, Revista de Antropología y Ciencias Sociales, Buenos Aires, № 6, abril de 2012, pp. 8 - 21. Recuperado el 20 de agosto de 2020 de: http://www.revistakula.com.ar/wp-content/uploads/2014/02/KULA6_1_ GESCO.pdf.

14. Hall, S. (2017). Estudios culturales 1983: una historia teorética. Buenos Aires: Paidós.

15. ----- (1980) Codificar/decodificar. En: Culture, Media y Lenguaje, London. Hutchinson. pp. 129-139. Traducción: Carlos Rusconi y Ariadna Cantú. Universidad Nacional de Río Cuarto: Departamento de Ciencias de la Comunicación.

16. Hoggart, R. (2013). La cultura obrera en la sociedad de masas.(título original: The uses of literacy). Traducido por: Julieta Barba y Silvia Jawerbaum.Buenos Aires: Siglo Veintiuno Editores.

17. O'Sullivan T., Hartley, J., Saunders D., Montgomery M, y Fiske J. (1995). Conceptos clave en comunicación y estudios culturales. Buenos Aires: Amorrortu.

18. Parkin, F. (1971/1978). Orden político y desigualdades de clase. Estratificación social de las sociedades capitalista y comunista. España: Debate.

19. Sarlo, B. (1994).Escenas de la vida posmoderna. Buenos Aires: Ariel.

20. ----- (1996). Instantáneas. Medios, ciudad y costumbres en el fin de siglo. Buenos Aires: Ariel.

21. Williams, R. (2001). Cultura y sociedad. Buenos Aires: Nueva Visión. 